\title{
Electron diffusion in polymer:fullerene bulk heterojunctions
}

\author{
Pieter A. C. Quist, Tom J. Savenije,* Juleon M. Schins, Jessica E. Kroeze, Paul A. Rijkers, and Laurens D. A. Siebbeles \\ Optoelectronic Materials Section, DelftChemTech, Faculty of Applied Sciences, Delft University of Technology, Julianalaan 136, \\ NL-2628 BL Delft, The Netherlands
}

(Received 10 November 2006; revised manuscript received 1 March 2007; published 15 May 2007)

\begin{abstract}
A method to study the bulk electron diffusion dynamics in poly(2-methoxy-5-(3', 7'-dimethyloctyloxy)1,4-phenylene-vinylene): 1-(3-methoxycarbonyl)-propyl-1-phenyl-(6,6) $\mathrm{C}_{61}$ (PCBM) blend films with varying PCBM content is presented. The blends are spin coated on top of dense smooth $\mathrm{TiO}_{2}$ and excited with a visible laser pulse. The photogenerated electrons on PCBM diffuse through the blend film until they reach the interface with $\mathrm{TiO}_{2}$, which serves to collect the electrons. The resulting change in conductance of $\mathrm{TiO}_{2}$ is measured using the time-resolved microwave conductivity technique. Theoretical analysis of the timedependent photoconductance gives the electron diffusion constant $\left(D_{e}\right)$ and the lower limit of the time for electrons to decay by trapping or recombination $\left(\tau_{e}\right) . D_{e}$ increases from $7.0 \times 10^{-13} \mathrm{~m}^{2} / \mathrm{s}$ at a weight fraction $\operatorname{PCBM}\left(W_{P C B M}\right)=0.11$ to $3.5 \times 10^{-10} \mathrm{~m}^{2} / \mathrm{s}$ at $W_{P C B M}=0.50$, while, interestingly, at $W_{P C B M}=0.75$, a smaller value of $1.2 \times 10^{-10} \mathrm{~m}^{2} / \mathrm{s}$ is found. The electron diffusion length increases with PCBM content. The presence of PCBM clusters in blends with a high PCBM content extends the time for electrons to decay, so that the lower limit of the electron diffusion length exceeds the film thickness of $100 \mathrm{~nm}$, which is required for sufficient light absorption in a photovoltaic device.
\end{abstract}

DOI: 10.1103/PhysRevB.75.195317

PACS number(s): 73.61.Ph, 72.20.Jv, 73.61.Wp, 73.50.Pz

\section{INTRODUCTION}

Currently, there is great interest in the development of photovoltaic devices based on blends of conjugated polymers with the fullerene derivative 1-(3-methoxycarbonyl)-propyl1-phenyl-(6,6) $\mathrm{C}_{61}(\mathrm{PCBM})$. In a pioneering study of Sariciftci et al., ${ }^{1}$ the photoinduced electron transfer from a conducting polymer to $\mathrm{C}_{60}$ was demonstrated. Because of the poor solubility of $\mathrm{C}_{60}$, better processable electron accepting compounds were developed, of which PCBM is applied mostly. The first photovoltaic devices based on blends of conjugated polymers and PCBM had only modest power efficiencies. 2,3 A breakthrough was achieved by Shaheen et al., ${ }^{4}$ who showed that the power efficiency could be improved to $2.5 \%$ under AM1.5 conditions, by using chlorobenzene as spin-coating solvent. This led to a number of investigations focusing on the effects of the spin-coating solvent on the morphology of the blend film and in turn on device performance..$^{5-11}$

Detailed investigations of the morphology and photophysical properties of blend films consisting of a conjugated polymer and PCBM have been carried out in order to find ways to enhance cell performance. ${ }^{7-10,12-21}$ Most studies involve blends of poly(2-methoxy-5-(3', 7'-dimethyloctyloxy)-1,4-phenylene-vinylene) (MDMOPPV) or poly(3-hexylthiophene) with PCBM. The influence of the blend morphology on charge generation, ${ }^{11,13,17,18}$ charge recombination, ${ }^{13,14,16-19,22}$ charge transport, $, 8,12,13,15,17-20,23$ and overall cell performance, ${ }^{7,8,13,21}$ has been studied. Particular attention has been paid to the influence of the PCBM content on device performance. For devices based on blends with MDMO-PPV, best performances have been reported for a weight fraction PCBM $\left(W_{P C B M}\right)$ of $0.80 .{ }^{4,8}$ This is a surprisingly high value, when it is realized that PCBM barely contributes to visible-light absorption. The high PCBM content is not needed to prevent fluorescent decay of the excitons that are produced in MDMO-PPV upon light absorption, since substantial quenching of fluorescence already occurs in blends with a $W_{P C B M}$ of only $0.02 .{ }^{11,24}$ It has been argued that a high PCBM content results in best device performance, due to formation of larger PCBM domains, leading to reduction of (geminate) charge recombination ${ }^{17,18}$ and/or efficient electron transport to the negative electrode. ${ }^{8}$ Moreover, a strong positive influence of the PCBM content on the hole mobility in MDMO-PPV has been found experimentally. ${ }^{12,13}$ Since in MDMO-PPV:PCBM blends the hole mobility is lower than the electron mobility, charge transport is limited by the holes. Using numerical modeling, it was shown by Mihailetchi et al. ${ }^{13}$ that for $W_{P C B M}$ up to 0.67 , cell performance improves due to an increase of the hole mobility with $W_{P C B M}$. For a further increase of $W_{P C B M}$ up to $\sim 0.80$, enhancement of the charge separation efficiency at the MDMO-PPV/PCBM interface leads to improvement of device performance. Only at even higher $W_{P C B M}$, reduced light absorption finally starts to limit the performance.

The morphological and charge transport studies on MDMO-PPV:PCBM blends mentioned above have yielded a wealth of useful information. However, more detailed insight into the relation between the PCBM content and device performance is lacking. In particular, the role PCBM clusters (PCBM-rich domains), appearing at $W_{P C B M}>0.60$ in charge generation and electron transport through a device, is not fully understood. Knowledge of the spatial distribution of PCBM in the blend that leads to optimum charge generation and transport may make it possible to reduce the PCBM content in a blend, leading to improved absorption of solar light and device performance. Hence, an important factor is the minimum PCBM content that is needed for efficient diffusion of electrons from their place of generation to the electrode. The present work aims to provide insight into the relation between PCBM content and electron diffusion and decay dynamics in blends with MDMO-PPV. This is 
(A)

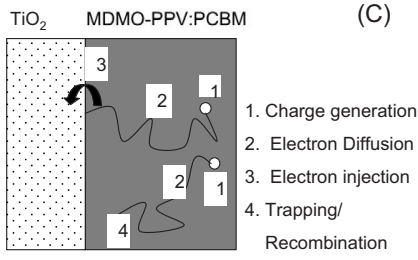

(B)

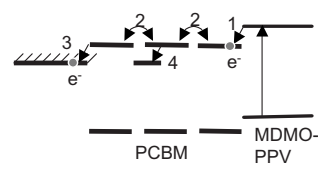

(D)

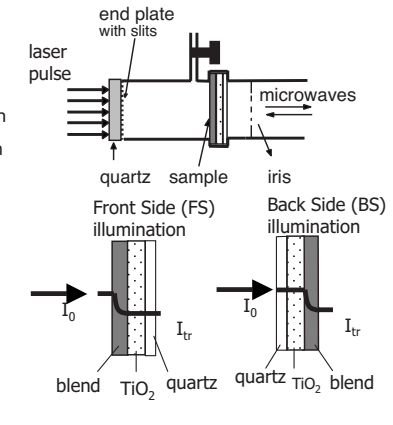

$\mathrm{TiO}_{2}$

FIG. 1. (A) Schematic representation of the processes occurring in an MDMO-PPV: $\mathrm{PCBM} \mathrm{TiO}_{2}$ film upon excitation with visible light. Electrons generated upon photoinduced charge separation in the blend film (1) diffuse (2) until they reach the interface with $\mathrm{TiO}_{2}$ where they are injected into the $\mathrm{TiO}_{2}$ conduction band (3). Part of the electrons recombine or are trapped (4). (B) Energy-level diagram and the processes depicted in panel (A). (C) Schematic representation of the measurement cell with the sample in the configuration for front side photoexcitation. (D) Schematic representation of the sample in the configurations for front side (FS) or back side (BS) photoexcitations together with the different excitation profiles.

achieved by using a bilayer configuration consisting of the bulk heterojunction on top of a $\mathrm{TiO}_{2}$ layer.

A schematic representation of the processes occurring after pulsed photoexcitation of the sample is given in Figs. 1(A) and 1(B). Absorption of visible light leads to formation of excitons in the polymer, which may diffuse to an MDMOPPV:PCBM interface, where they can dissociate to produce electrons in PCBM and holes in the polymer [process (1)]. The electrons can diffuse through the blend layer by hopping via PCBM molecules or aggregates and may reach the interface between the blend layer and the $\mathrm{TiO}_{2}$ layer [process (2)]. At the interface, electrons can be injected into the conduction band of $\mathrm{TiO}_{2}$ [process (3)]. Electron diffusion through the blend layer occurs in competition with decay by recombination with holes in MDMO-PPV and trapping which ends diffusion [process (4)]. Injection from PCBM into $\mathrm{TiO}_{2}$ is thermodynamically favorable, since the electron affinity of $\mathrm{TiO}_{2}(4.2 \mathrm{eV})$ is about $0.5 \mathrm{eV}$ larger than that of PCBM. ${ }^{25,26}$ In the dense $\mathrm{TiO}_{2}$ layers used, the ac electron mobility is approximately 2 orders of magnitude higher than in PCBM. ${ }^{18}$ Hence, electron injection into $\mathrm{TiO}_{2}$ results in an increase of the photoconductance with time. In this way, the $\mathrm{TiO}_{2}$ layer serves to monitor electron diffusion from the position of photogeneration in the MDMO-PPV:PCBM layer to the $\mathrm{TiO}_{2}$ layer. The electron diffusion constant in the MDMO-PCBM blend film and the time before decay of the electron by trapping or recombination could be obtained from theoretical analysis of the time-dependent photoconductance due to accumulation of electrons in $\mathrm{TiO}_{2}$. Knowledge of both the electron diffusion constant together with the time for decay by trapping or recombination enables the determination of the electron diffusion length. The influence of blend morphology on the electron diffusion dynamics and the implications for photovoltaic devices based on an MDMO:PCBM blend are discussed.

\section{EXPERIMENT}

\section{Sample preparation}

MDMO-PPV (synthesized via the Vanderzande route ${ }^{27}$ $M_{w}=57 \times 10^{3} \mathrm{~g} \mathrm{~mol}^{-1}$ ) was obtained from TNO-Eindhoven, The Netherlands, and mixed with PCBM, purchased from Syncom (Groningen, The Netherlands). 0.8 wt $\%$ polymer solutions in chlorobenzene were prepared to which appropriate amounts of PCBM were added to obtain PCBM weight ratios (weight PCBM/total weight) of $0.11,0.25,0.50$, and 0.75 . The solutions were stirred for at least $24 \mathrm{~h}$ at $50{ }^{\circ} \mathrm{C}$ before blend films were spin coated onto $\sim 1$-mm-thick, 12 $\times 25 \mathrm{~mm}^{2}$ substrates, using a spin speed of $1500 \mathrm{rpm}$ for $\sim 60 \mathrm{~s}$ at room temperature. The substrates used are quartz plates (ESCO Products) onto which an $\sim 100$-nm-thick smooth, anatase $\mathrm{TiO}_{2}$ layer is deposited by means of chemical-vapor deposition (Everest Coatings, Delft, The Netherlands). Sample preparation took place under $\mathrm{N}_{2}$ atmosphere with an oxygen concentration $<10$ ppm humidity and $<1 \mathrm{ppm}$. The thickness of the blend films $L$ was determined using a step profiler with a stylus force of $0.03 \mathrm{mg}$ and a tip radius of $12 \mu \mathrm{m}$ (Dektak 8, Veeco).

\section{Optical characterization}

A Perkin Elmer Lambda 900 ultraviolet-visible-nearinfrared spectrophotometer equipped with an integrating sphere (Lab sphere) was used to measure the fraction of incident light reflected $\left(F_{R}\right)$ and transmitted $\left(F_{T}\right)$ by the samples, from which the optical absorption $(O D)$ and attenuation $\left(F_{A}=1-F_{T}-F_{R}\right)$ spectra were determined. ${ }^{28}$ From the optical absorption and thickness measurements, wavelengthdependent absorption coefficients $\alpha(\lambda)$ can be determined using $\alpha(\lambda)=(\ln 10) O D(\lambda) / L$.

\section{Flash-photolysis time-resolved microwave conductivity (FP-TRMC)}

Immediately after preparation, the samples were mounted in an $X$-band microwave cavity [see Fig. 1(C)]. For photoexcitation at $\lambda=510$ or $600 \mathrm{~nm}$, a Nd-doped yttrium aluminium garnet laser (Infinity 15-30, Coherent) was used to pump an optical parametric oscillator, yielding $3 \mathrm{~ns}$ full width at half maximum. For excitation at $\lambda=300 \mathrm{~nm}$ a second-harmonic generation crystal was used. Any photoinduced change in the conductance $(\Delta G)$ of the sample was monitored as a relative change in the microwave power $(\Delta P / P)$ reflected by the cavity as described previously. ${ }^{28}$ The results of the TRMC measurements are expressed in terms of $\Delta G(t)$, using

$$
\Delta G(t)=-\frac{1}{K} \frac{\Delta P(t)}{P},
$$

where $K$ is the sensitivity factor of the cavity containing the sample and amounts to $5 \times 10^{4} \Omega$. The product $\eta \Sigma \mu$, in which $\Sigma \mu$ is the sum of the mobility of a positive and a negative charge carrier and $\eta$ is defined as the ratio of the number of charge carrier pairs at the maximum of the $\Delta G$ transient and the total number of incident photons from the laser pulse, can be calculated by 


$$
\eta \Sigma \mu=\frac{\Delta G_{\max }}{\beta e I_{0}}
$$

where $\beta=2.1$ corresponds to the ratio of the rectangular dimensions of the waveguide and $e$ is the elementary charge.

The incident photon to charge separation efficiency (IPCSE) is defined such that it corresponds to the number of electrons injected from the blend into $\mathrm{TiO}_{2}$ normalized to the number of incident photons. The IPCSE is obtained using

$$
\operatorname{IPCSE}=\frac{\eta \Sigma \mu_{v i s}}{\left(\eta \Sigma \mu_{300 \mathrm{~nm}} / F_{A 300 \mathrm{~nm}}\right)} \times 100 \%,
$$

where $\eta \Sigma \mu_{v i s}$ is the $\eta \Sigma \mu$ signal obtained upon excitation in the visible, while $\eta \Sigma \mu / F_{A}(300 \mathrm{~nm})$ is the $\eta \Sigma \mu / F_{A}$ signal obtained upon excitation of the $\mathrm{TiO}_{2}$ layer at $\lambda=300 \mathrm{~nm}$. Equation (3) can be justified as follows. Since the mobility of electrons in $\mathrm{TiO}_{2}, \mu_{e}\left(\mathrm{TiO}_{2}\right)$, is much higher than that of charge carriers in the blend film, it follows that $\Sigma \mu$ $\approx \mu_{e}\left(\mathrm{TiO}_{2}\right)$. At $300 \mathrm{~nm}$, direct-band-gap excitation of $\mathrm{TiO}_{2}$ takes place with a quantum efficiency of almost unity; i.e., $\eta / F_{A}(300 \mathrm{~nm}) \approx 1$. Therefore, $\eta \Sigma \mu / F_{A}(300 \mathrm{~nm})$ is a good estimate for the mobility of electrons in $\mathrm{TiO}_{2}$. The value of $\mu_{e}\left(\mathrm{TiO}_{2}\right)$ was determined for each $\mathrm{TiO}_{2}$ substrate used.

\section{RESULTS AND DISCUSSION}

\section{Experimental results}

In what follows, blend film samples are denoted using the annotation $\mathrm{PPV}: \mathrm{PCBM}_{x} / y$ with $x$ the weight fraction of PCBM in the film blended with MDMO-PPV and $y$ the substrate onto which the film is spin coated (quartz alone or $\mathrm{TiO}_{2}$ on quartz).

\section{Optical properties and transient photoconductance}

Figure 2 shows spectra of the absorption coefficients of a $\mathrm{PPV}: \mathrm{PCBM}_{0.50} /$ quartz bilayer and of the individual compounds on quartz. The $O D$ spectrum of a bare $\mathrm{TiO}_{2}$ film is given against the right-hand axis. Comparison of the spectra shows that the absorption at $300 \mathrm{~nm}$ is mainly due to $\mathrm{TiO}_{2}$, while above $400 \mathrm{~nm}$ absorption is due to the organic blend layer. In the TRMC experiments, $\mathrm{TiO}_{2}$ is almost selectively excited at $\lambda=300 \mathrm{~nm}$, while at $\lambda=510$ or $600 \mathrm{~nm}$ only the blend is excited.

Figure 3(A) shows photoconductance transients for photoexcitation at $510 \mathrm{~nm}$ for an $\mathrm{MDMO}-\mathrm{PPV} / \mathrm{TiO}_{2}$ bilayer and for a PPV: $\mathrm{PCBM}_{0.50} / \mathrm{TiO}_{2}$ bilayer. In addition, the photoconductance obtained on photoexcitation of the $\mathrm{PPV}: \mathrm{PCBM}_{0.50} / \mathrm{TiO}_{2}$ bilayer at $300 \mathrm{~nm}$ is shown. Clearly, the transients exhibit very different kinetics. The temporal shape of the rise of the transients for the $\mathrm{PPV}: \mathrm{PCBM}_{0.50} / \mathrm{TiO}_{2}$ film on photoexcitation at $300 \mathrm{~nm}$ and for the MDMO-PPV/ $\mathrm{TiO}_{2}$ bilayer on photoexcitation at $510 \mathrm{~nm}$ is determined by the $3 \mathrm{~ns}$ laser-pulse duration and the $18 \mathrm{~ns}$ response time of the microwave cavity. This is demonstrated by the agreement between the rise of the photoconductance of the PPV:PCBM $\mathrm{P}_{0.50} / \mathrm{TiO}_{2}$ bilayer excited at $300 \mathrm{~nm}$ and the full line, which is the integrated intensity

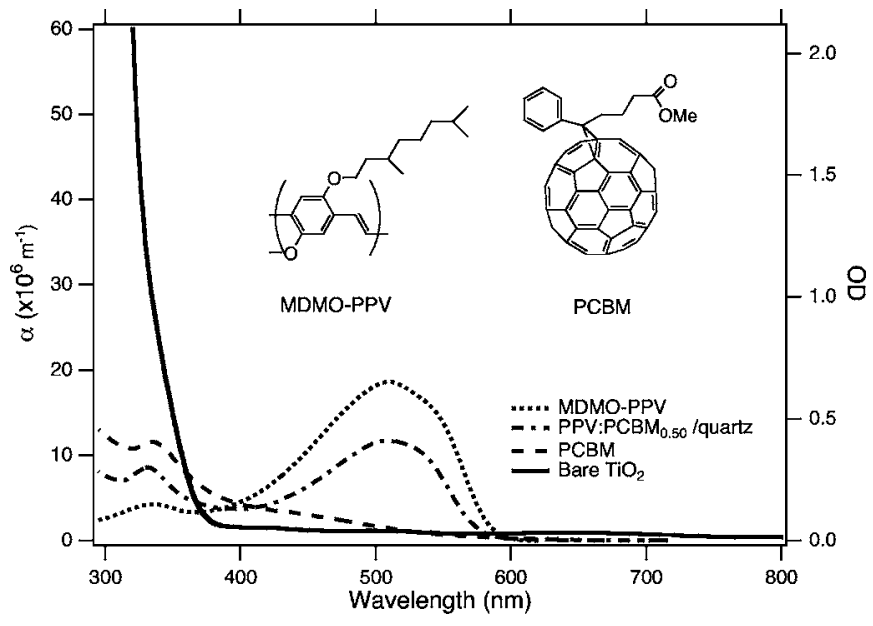

FIG. 2. Absorption coefficients of MDMO-PPV on quartz (dotted; film thickness $L=359 \mathrm{~nm}$ ), of a PPV: $\mathrm{PCBM}_{0.50} /$ quartz blend film (dot dash; $L=75 \mathrm{~nm}$ ), and of PCBM (dashed). The absorption spectrum of a bare $\mathrm{TiO}_{2}$ film on quartz (full line) is given on the right-hand axis for comparison. The molecular structures of MDMO-PPV and PCBM are also shown.

during the laser pulse convoluted with 18 ns response of the cavity.

The photoconductance of the PPV: $\mathrm{PCBM}_{0.50} / \mathrm{TiO}_{2}$ film obtained on excitation at $300 \mathrm{~nm}$ is due to band-gap excitation of electrons in $\mathrm{TiO}_{2}$. The relatively fast decay of the photoconductance is due to recombination of the electrons and holes that are formed within the semiconductor film. The photoconductance for the MDMO-PPV/ $\mathrm{TiO}_{2}$ bilayer for excitation at $510 \mathrm{~nm}$ is attributed to electrons in $\mathrm{TiO}_{2}$, which are produced by dissociation of excitons at the interface between MDMO-PPV and $\mathrm{TiO}_{2}$. The photoconductance is long lived $(>100 \mu \mathrm{s})$, due to the slow recombination of electrons in the $\mathrm{TiO}_{2}$ layer with holes in MDMO-PPV. These results are in full agreement with those reported earlier. ${ }^{29}$ The photoconductance transient for the PPV: $\mathrm{PCBM}_{0.50} / \mathrm{TiO}_{2}$ bilayer obtained on excitation at $510 \mathrm{~nm}$ exhibits a much slower increase than the instrument limited rise of the transient for the MDMO-PPV/ $\mathrm{TiO}_{2}$ bilayer. Hence, the photoconductance in the PPV: $\mathrm{PCBM}_{0.50} / \mathrm{TiO}_{2}$ bilayer is not due to fast production of electrons in $\mathrm{TiO}_{2}$ by dissociation of excitons at the interface between MDMO-PPV and $\mathrm{TiO}_{2}$. Previous work has demonstrated that visible excitation of PPV: $\mathrm{PCBM}_{x} /$ quartz ${ }^{18}$ or bare $\mathrm{TiO}_{2}$ layers ${ }^{29,30}$ alone results in much smaller and faster (response-time limited) photoconductance signals. Therefore, the relatively slow rise of the photoconductance for the PPV: $\mathrm{PCBM}_{0.50} / \mathrm{TiO}_{2}$ bilayer is attributed to diffusion of electrons from the blend film into the $\mathrm{TiO}_{2}$ layer (see Fig. 1). This causes the photoconductance to increase with time, since the mobility of electrons in $\mathrm{TiO}_{2}$ is much higher than in the organic blend film.

To confirm the assignment of the increase of the photoconductance during the time of diffusion of electrons from the blend film into the $\mathrm{TiO}_{2}$ layer, the initial spatial distribution of the photoexcitations in the blend film was varied, as illustrated in Fig. 1(D). Excitations can be produced predominantly away from or near the interface between the or- 

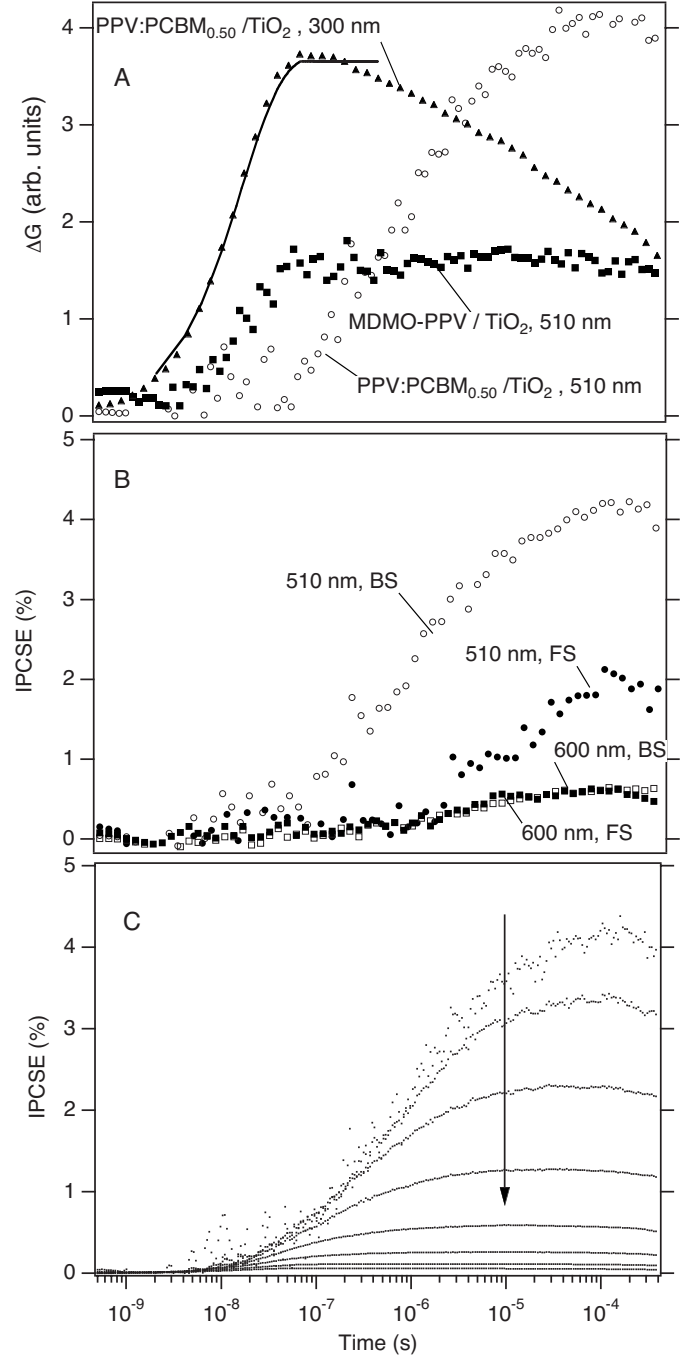

FIG. 3. (A) Photoconductance transients on excitation at $510 \mathrm{~nm}$ of a pure MDMO-PPV film on $\mathrm{TiO}_{2}$ (squares) and of a $\mathrm{PPV}: \mathrm{PCBM}_{0.50} / \mathrm{TiO}_{2}$ film (circles). Excitation was from the back side with an intensity of $3.9 \times 10^{11}$ and $1.5 \times 10^{11}$ photons/ $\mathrm{cm}^{2} /$ pulse, respectively. In addition, the photoconductance of the $\mathrm{PPV}: \mathrm{PCBM}_{0.50} / \mathrm{TiO}_{2}$ film for excitation at $300 \mathrm{~nm}$ (triangles) is also shown. The full line gives the integrated intensity during the laser pulse convoluted with the $18 \mathrm{~ns}$ response time of detection. (B) IPCSE transients on excitation of the PPV : $\mathrm{PCBM}_{0.50} / \mathrm{TiO}_{2}$ film under conditions as indicated in the figure. Note that the two transients on excitation at $600 \mathrm{~nm}$ coincide almost completely. (C) IPCSE transients on excitation of the PPV: $\mathrm{PCBM}_{0.50} / \mathrm{TiO}_{2}$ blend film at different excitation intensities. The pulse-integrated intensities were (increasing in the direction of the arrow) $1.5 \times 10^{11}, 5.3$ $\times 10^{11}, 1.6 \times 10^{12}, 5.0 \times 10^{12}, 1.8 \times 10^{13}, 5.8 \times 10^{13}, 2.1 \times 10^{14}$, and $6.8 \times 10^{14}$ photons $/ \mathrm{cm}^{2} /$ pulse.

ganic blend layer and $\mathrm{TiO}_{2}$, by illumination of the sample from the side of the blend layer [front side (FS) excitation or via back side (BS) excitation], respectively. The excitation density can be reduced by variation of the excitation wavelength from 510 to $600 \mathrm{~nm}$ due to the much smaller absorption coefficient of MDMO-PPV and PCBM at the longer wavelength [see Fig. 2]. Figure 3(B) shows IPCSE transients, as defined by Eq. (3), for the PPV:PCBM ${ }_{0.50} / \mathrm{TiO}_{2}$ bilayer obtained by FS or BS excitation at 510 or $600 \mathrm{~nm}$. At $510 \mathrm{~nm}$, FS photoexcitation leads to a much slower increase of the photoconductance with time than for BS excitation. In addition, the IPCSE reached at longer times is more than a factor of 2 lower for FS excitation than for BS excitation. These findings can be qualitatively understood as follows. Due to the high absorption coefficient at $510 \mathrm{~nm}$, excitations are generated in the organic blend layer in a region near the side where the laser pulse enters the sample. Hence, for FS excitation charge carriers are produced significantly further away from the interface with $\mathrm{TiO}_{2}$ than for BS photoexcitation. As a result, for FS excitation electrons need to diffuse over a longer distance through the blend layer to reach the $\mathrm{TiO}_{2}$ layer, than in the case of BS excitation. This causes the increase of the photoconductance to be slower for FS excitation. Since electrons in the blend film can also become trapped or recombine, the longer period needed for diffusion upon FS excitation also results in a lower IPCSE signal. At $600 \mathrm{~nm}$, the absorption coefficient of the blend film is considerably smaller, which results in lower IPCSE values [see Fig. 3(B)]. The IPCSE transients in Fig. 3(B) for FS and BS excitations at $600 \mathrm{~nm}$ are similar. This is due to the relatively small absorption coefficient at $600 \mathrm{~nm}$, which causes a close to constant absorption of light as a function of depth in the blend layer. Hence, both FS and BS excitations at $600 \mathrm{~nm}$ lead to an almost homogeneous initial distribution of charge carriers in the blend film. Consequently, the kinetics of electron diffusion from the blend film to the $\mathrm{TiO}_{2}$ layer is similar for FS and BS excitations.

The data in Fig. 3(C) show the effect of the laser-pulse intensity on the IPCSE for BS excitation of the PPV : $\mathrm{PCBM}_{0.50} / \mathrm{TiO}_{2}$ blend film at $510 \mathrm{~nm}$. The IPCSE reached at longer times decreases as the intensity becomes higher. This is attributed to enhanced second-order charge recombination at higher intensities, leading to a decreased number of charge carriers per incident photon reaching the interface with $\mathrm{TiO}_{2}$. The fact that the transients for the two lowest intensities are different indicates that even at these low intensities second-order recombination plays a significant role. Measurements at lower laser-pulse intensities were hampered by a too small signal-to-noise ratio.

\section{Effect of PCBM content on IPCSE}

Figure 4 shows the effects of the PCBM content on the IPCSE transients obtained on FS or BS excitation at $510 \mathrm{~nm}$ for low laser-pulse intensities. Both for FS and BS excitations, the IPCSE value reached at longer times increases with $W_{P C B M}$. The mean distance between PCBM molecules decreases with $W_{P C B M}$, resulting in a higher electron-hopping rate between PCBM molecules. The higher electron diffusion constant together with the improvement of electron percolation paths leads to enhanced electron diffusion toward $\mathrm{TiO}_{2}$, rather than electron decay by recombination or trapping. The IPCSE values are higher for BS excitation than for FS excitation. As discussed above, this is due to the fact that for BS excitation, electrons are initially generated closer to the interface between the blend film and the $\mathrm{TiO}_{2}$ layer. The IPCSE transients in Figs. 4(A) and 4(B) for lower $W_{P C B M}$ values exhibit an initial rise on a short time scale in addition 
to the increase at longer times. The initial rise in Figs. 4(A) and 4(B) occurs on a time scale, which is determined by the $18 \mathrm{~ns}$ response time of the microwave cavity. This component in the IPCSE transient is attributed to fast production of electrons in $\mathrm{TiO}_{2}$, by dissociation of excitons that are initially photogenerated in MDMO-PPV near the interface with $\mathrm{TiO}_{2}$. At lower $W_{P C B M}$, this process of direct electron injection from MDMO-PPV into $\mathrm{TiO}_{2}$ competes with exciton dissociation at an interface with PCBM. The absence of the initial rise at shorter times in the transient in. 4(C) shows that for $W_{P C B M}=0.50$ excitons decay by electron transfer to PCBM, followed by electron diffusion toward the $\mathrm{TiO}_{2}$ layer.

The transients for the PPV: $\mathrm{PCBM}_{0.75} / \mathrm{TiO}_{2}$ blend film in. 4(D) show a contribution to the signal, which rises on a time scale determined by the response time and decays on a time scale of $\sim 1 \mu \mathrm{s}$. This contribution is similar in shape and magnitude as TRMC signals obtained in previous work on PPV:PCBM blend samples with $W_{P C B M}=0.75$ spin coated onto quartz substrates. ${ }^{17}$ The PPV: $\mathrm{PCBM}_{0.75} / \mathrm{TiO}_{2}$ blend film contains PCBM clusters, ${ }^{5,8}$ in contrast to the films with $W_{P C B M} \leqslant 0.50$. The signal on shorter times is attributed to mobile electrons moving within the PCBM clusters, analogous to previous work, ${ }^{17,18}$ and not to electrons in $\mathrm{TiO}_{2}$. Only the IPCSE signal, which increases at times exceeding $1 \mu \mathrm{s}$, is attributed to electron diffusion through the blend layer followed by electron injection into $\mathrm{TiO}_{2}$.

\section{Theoretical analysis of IPCSE transients}

\section{Theoretical model}

Quantitative information about the electron diffusion coefficient $\left(D_{e}\right)$ and the average time before decay of an electron by trapping or recombination $\left(\tau_{e}\right)$ was obtained by theoretical analysis of the IPCSE transients of Fig. 4. The timedependent number of electrons injected into $\mathrm{TiO}_{2}$ can be described by a theoretical model for electron diffusion, as outlined in the Appendix. In this model, the initial distribution of photogenerated electrons is taken to be a LambertBeer profile. After instantaneous photogeneration, the electrons are assumed to perform a diffusive motion. The electrons can cross the interface between the blend layer and $\mathrm{TiO}_{2}$, which is assumed to occur in competition with firstorder decay within the blend film. Time integration of the flux of charges diffusing across the interface between the blend and $\mathrm{TiO}_{2}$ gives the accumulated number of injected electrons in $\mathrm{TiO}_{2}$ as a function of time. Recombination of electrons in $\mathrm{TiO}_{2}$ with holes in the organic blend is neglected. The latter is justified by the very long chargeseparated lifetime observed for the MDMO-PPV/TiO 2 bilayer [see Fig. 3(A)]. The number of electrons traversing the interface between the blend and $\mathrm{TiO}_{2}$, normalized to the number of incident photons, as function of time is given by

$$
N(t)=\left(\frac{2}{1-e^{-m \alpha L}}\right) \sum_{k=0}^{\infty}\left\{\frac{\left(\frac{(-1)^{k}}{\pi(k+1 / 2)}+\frac{e^{-m \alpha L}}{m \alpha L}\right)\left(1-e^{-\left\{1 / \tau_{e}+[\pi(k+1 / 2) / L]^{2} D_{e}\right\} t}\right)}{\left[1+\left(\frac{\pi(k+1 / 2)}{\alpha L}\right)^{2}\right]\left[1+\frac{1}{D_{e} \tau_{e}}\left(\frac{L}{\pi(k+1 / 2)}\right)^{2}\right]}\right\},
$$

where $\alpha$ is the wavelength-dependent absorption coefficient of the blend film, $\tau_{e}$ is the time before decay of an electron by trapping or recombination within the blend, and $L$ is the thickness of the blend film. For FS excitation the parameter $m=1$, while for BS excitation $m=-1$. From $N(t)$, the IPCSE can be calculated by

$$
\operatorname{IPCSE}(t)=\left(1-F_{R}\right) \varphi N(t) .
$$

The factor $F_{R}$ is the fraction of incident light that is reflected. The scaling factor $\varphi$ accounts for (a) the quantum efficiency for free charge generation and (b) the efficiency for electron injection into the conduction band of $\mathrm{TiO}_{2}$ on encounter of an electron at the interface between the blend film and $\mathrm{TiO}_{2}$.

Note that in the theoretical model, second-order charge recombination is incorporated in the time for first-order decay of the electrons $\left(\tau_{e}\right)$. This means that recombination is treated as a first-order process equivalent to trapping of electrons. This approach provides an adequate description of the transients at the lowest laser intensities used, as evidenced by the finding that transients obtained at a somewhat higher intensity could be fitted using the same $D_{e}$ and $\varphi$ and a somewhat shorter $\tau_{e}$ as also shown in the Appendix. Hence, the found $\tau_{e}$ is a lower limit for the actual lifetime.

As already discussed above, the transients in Figs. 4(A) and 4(B) contain a contribution to the IPCSE signal on shorter times, due to direct electron injection into $\mathrm{TiO}_{2}$ from excitons in MDMO-PPV. This contribution to the IPCSE transients is taken into account by addition of an instantaneously produced contribution, $N_{\text {excit }}$, giving

$$
\operatorname{IPCSE}(t)=\left(1-F_{R}\right)\left[N_{\text {excit }}+\varphi N(t)\right] .
$$

As mentioned above, the signal in the transients of the PPV: $\mathrm{PCBM}_{0.75} / \mathrm{TiO}_{2}$ blend film [Fig. 4(D)] at times below $1 \mu$ s is due to mobile electrons moving within large PCBM clusters and is not due to electron diffusion through the blend followed by injection of electrons into $\mathrm{TiO}_{2}$. Therefore, this contribution is ignored when comparing the experimental data with the theoretical model. The wavelength-dependent absorption coefficient $\alpha(\lambda)$ is calculated from $\alpha(\lambda)$ $=(\ln 10) O D(\lambda) / L$. For each weight fraction, the fit param- 

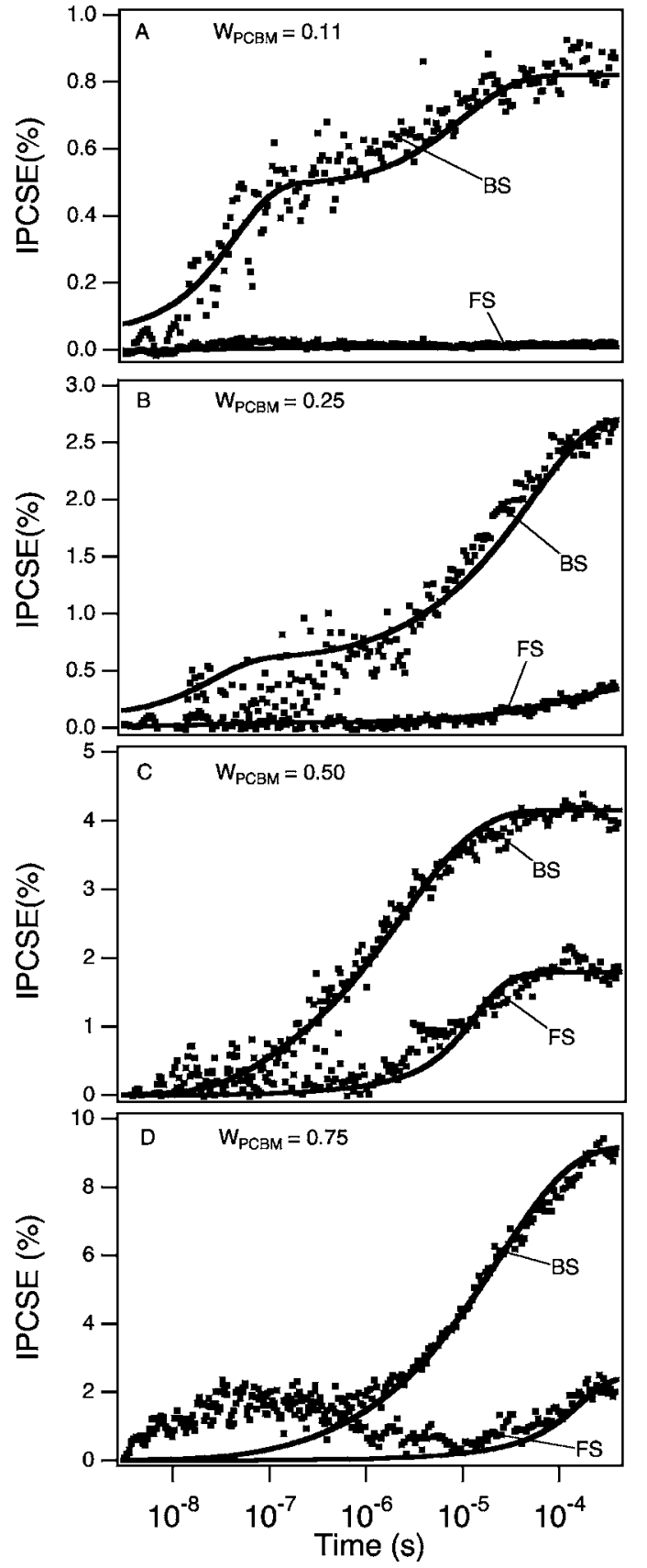

FIG. 4. IPCSE transients on excitation at $510 \mathrm{~nm}$ of MDMOPPV:PCBM blend films with (A) $W_{P C B M}=0.11$, (B) $W_{P C B M}=0.25$, (C) $W_{P C B M}=0.50$, and (D) $W_{P C B M}=0.75$, with an intensity of $(2 \pm 1) \times 10^{11}$ photons $/ \mathrm{cm}^{2} /$ pulse. Transients are shown for both FS and BS excitations, as indicated. The lines are fits to the data according to Eq. (6). In panel (A), the FS signal almost coincides with the horizontal axis.

eters $D_{e}, \tau_{e}$, and $\varphi$ were adjusted to obtain optimum agreement between the transients calculated using Eqs. (5) and (6) and the measured data for a series of transients obtained on FS or BS excitation at various wavelengths. The calculated transients were convoluted with the $18 \mathrm{~ns}$ response time of the microwave cavity. The fit results are represented by the full lines in Fig. 4 and the values of the fit parameters are presented in Table I. The results in Fig. 4 show that the
TABLE I. Film properties and simulation parameters.

\begin{tabular}{lcccc}
\hline \hline & \multicolumn{4}{c}{$W_{P C B M}$} \\
\cline { 2 - 5 } & 0.11 & 0.25 & 0.50 & 0.75 \\
\hline$O D$ & 2.04 & 1.19 & 1.37 & 1.55 \\
$L(\mathrm{~nm})$ & 159 & 124 & 143 & 422 \\
$D_{e}\left(\mathrm{~m}^{2} / \mathrm{s}\right)$ & $7.0 \times 10^{-13}$ & $2.0 \times 10^{-12}$ & $3.5 \times 10^{-10}$ & $1.2 \times 10^{-10}$ \\
$\tau_{e}(\mathrm{~s})^{\mathrm{a}}$ & $2.0 \times 10^{-5}$ & $1.5 \times 10^{-4}$ & $1.1 \times 10^{-5}$ & $1.4 \times 10^{-4}$ \\
$\Lambda_{e}(\mathrm{~nm})^{\mathrm{a}, \mathrm{b}}$ & 4 & 17 & 62 & 130 \\
$\varphi$ & 0.05 & 0.09 & 0.08 & 0.21 \\
\hline \hline
\end{tabular}

The values given represent the lower limit.

${ }^{\mathrm{b}}$ The parameter $\Lambda_{e}$ is the electron diffusion length, defined by $\Lambda_{e}$ $=\sqrt{D_{e} \tau_{e}}$.

experimental data are very well reproduced by the theoretical model.

\section{Effect of PCBM content on $D_{e}, \tau_{e}, \varphi$, and the electron diffusion length}

The data in Table I show that the electron diffusion constant $D_{e}$ increases with the content PCBM on going from $W_{P C B M}=0.11$ to $W_{P C B M}=0.50$ and then decreases on going to $W_{P C B M}=0.75$. The increase of $D_{e}$ with $W_{P C B M}$ up to 0.50 will be due to the higher rate for hopping of electrons as a result of the smaller distance between PCBM molecules. In addition, the diffusion constant can increase on higher PCBM concentrations due to enhanced formation of electron percolation pathways via the network of PCBM molecules and/or nanocrystals. ${ }^{8}$

Remarkably, for the PPV: $\mathrm{PCBM}_{0.75} / \mathrm{TiO}_{2}$ blend film $D_{e}$ is about three times smaller than that for the PPV : $\mathrm{PCBM}_{0.50} / \mathrm{TiO}_{2}$ blend film. As is explained below, this reduction of $D_{e}$ could be due to the presence of PCBM clusters in films with such a high PCBM content. ${ }^{17}$ In Ref. 17 a lower limit of $4 \times 10^{-2} \mathrm{~cm}^{2} / \mathrm{Vs}$ is found for the microwave mobility $(8.5 \mathrm{GHz})$ of electrons moving within a PCBM cluster. The corresponding value for $D_{e}, 1 \times 10^{-7} \mathrm{~m}^{2} / \mathrm{s}$, is more than 800 times larger than the value found for the $\mathrm{PPV}: \mathrm{PCBM}_{0.75} / \mathrm{TiO}_{2}$ blend film in this work. This observation eliminates the possibility that electrons in the PCBM clusters are directly injected into the conduction band of $\mathrm{TiO}_{2}$, since in that case a faster growing contribution in the IPCSE would have been observed. Therefore, for transport to $\mathrm{TiO}_{2}$, electrons have to (re)enter the MDMO-PPV:PCBM matrix. The low $D_{e}$ observed can then be explained by a relatively low rate constant for electron transfer from a PCBM cluster to the matrix, which is amenable if, as suggested above, the energy of the lowest unoccupied molecular orbital level of a PCBM cluster is lower than that of a single PCBM molecule and/or PCBM nanocrystal in the matrix.

The influence of $W_{P C B M}$ on the time before decay of the electrons by trapping or recombination is not straightforward. After an initial increase by almost an order of magnitude on going from $W_{P C B M}=0.11$ to $W_{P C B M}=0.25$, this time decreases again on going to $W_{P C B M}=0.50$. The maximum 
value of $\tau_{e}$ at $W_{P C B M}=0.25$ may result from the opposite effects of the PCBM content on electron trapping and charge recombination. In the absence of PCBM clusters (i.e., $\left.W_{P C B M}<0.60\right)$, the rate of charge recombination is expected to increase with $W_{P C B M}$ as a consequence of an increase of the mobility of charge carriers, as reported by Mihailetchi et al. ${ }^{13}$ The increase of the time for the electron to decay on going from $W_{P C B M}=0.11$ to 0.25 may be due to a dominant effect of a reduction of trapping. For a further increase to $W_{P C B M}=0.50$, the enhancement of the rate of charge recombination then dominates, which leads to a shorter time to decay.

Interestingly, for electrons in the PPV: $\mathrm{PCBM}_{0.75} / \mathrm{TiO}_{2}$ blend film, $\tau_{e}$ is more than an order of magnitude higher than for the PPV: $\mathrm{PCBM}_{0.50} / \mathrm{TiO}_{2}$ blend film. In the $\mathrm{PPV}: \mathrm{PCBM}_{0.75} / \mathrm{TiO}_{2}$ blend, charge recombination is suppressed due to electrons entering larger PCBM clusters followed by electron diffusion away from the positive counter charge in PPV, as discussed earlier. ${ }^{17,18}$

The diffusion constants in Table I can be related to the dc electron mobility by using the Einstein relation $\mu=e D_{e} / k_{B} T$. For $W_{P C B M} \leqslant 0.50$, the mobilities obtained in this way are close to literature data. ${ }^{13,20,31}$ Remaining differences between the present work and literature may in part be due to the dependence of the electron mobility on the concentration of charges. ${ }^{13}$ While in the present work the mobility decreases on going from $W_{P C B M}=0.50$ to 0.75 , the mobilities reported in Refs. 13 and 29 continue to increase. An explanation for the different behavior may be that the applied voltage bias in the work of Refs. 13 and 29 reduces the energetic barrier between electrons in PCBM clusters and on isolated PCBM molecules. This would result in an enhanced electron transfer rate from PCBM clusters toward the matrix and in turn a higher mobility.

Table I shows that the factor $\varphi$ varies between 0.05 and 0.09 for blend films with $W_{P C B M} \leqslant 0.50$ and amounts to 0.21 for the PPV: $\mathrm{PCBM}_{0.75} / \mathrm{TiO}_{2}$ blend film. As mentioned above, the factor $\varphi$ takes into account the quantum yield for electron generation in the MDMO-PPV:PCBM blend and the efficiency of electron injection into $\mathrm{TiO}_{2}$ upon arrival of an electron at the interface. Savenije et al. ${ }^{18}$ have estimated the quantum yield for generation of charge carriers in MDMO-PPV:PCBM blend films with PCBM weight fractions between 0.2 and 0.6 to be $\sim 0.3$. This implies that that $16 \%-30 \%$ of the electrons undergo transfer into $\mathrm{TiO}_{2}$ upon arrival at the interface. For blend films with $W_{P C B M}$ $>0.60$, Savenije et al. ${ }^{18}$ estimated the quantum yield of mobile charge carriers to be close to 1, giving an interfacial electron transfer efficiency of approximately $21 \%$. The fact that this value is within the range of $16 \%-30 \%$ found above for the charge-transfer efficiency from blend films with a PCBM content $\leqslant 0.50$ agrees with the picture discussed above that electron injection in the PPV: $\mathrm{PCBM}_{0.75} / \mathrm{TiO}_{2}$ blend film takes place from the matrix rather than from PCBM clusters.

The lower limit of the electron diffusion length, defined as $\Lambda_{e}=\sqrt{D_{e} \tau_{e}}$, is added to Table I for the different blends investigated. From these values, it is clear that the electron diffusion length increases with the PCBM content. The value of $\Lambda_{e}$ in the PPV: $\mathrm{PCBM}_{0.50} / \mathrm{TiO}_{2}$ blend film amounts to $62 \mathrm{~nm}$, which is not very different from the value of $45 \mathrm{~nm}$ found earlier as the electron diffusion length in the matrix of MDMO-PPV:PCBM blends spin coated from toluene. ${ }^{17}$

At low-intensity illumination, the concentration of charge carriers in our samples directly after the laser pulse is in the same order of magnitude as in solar cells working at maximum power point. Moreover, near the maximum power point, diffusion is the main charge transport mechanism in these devices. ${ }^{14}$ Therefore, the electron transport through the PPV: $\mathrm{PCBM}_{0.75} / \mathrm{TiO}_{2}$ blend film, as described above, can be considered to be representative for that operative in optimized working devices. The optimum $W_{P C B M}$ value found for MDMO-PPV:PCBM photovoltaic devices amounts to 0.80 .4 In order to absorb sufficient solar light, blend films with such a high PCBM content have a thickness of at least $100 \mathrm{~nm}$. The data in Table I show that the electron diffusion length exceeds this typical film thickness only for the highest PCBM content studied in the present work, i.e., $W_{P C B M}$ $=0.75$. According to van Duren et al.,${ }^{8}$ a high PCBM content is needed for the formation of PCBM clusters, which mediate electron transport to the electrodes. The results of the present work indicate that PCBM clusters serve not so much as to transport electrons (faster) toward the electrodes, but rather to reduce the recombination rate. The longer time for electrons to decay in blends with a high PCBM content leads to a diffusion length comparable to the typical film thickness used in devices.

\section{CONCLUSIONS}

The effect of PCBM content on electron diffusion dynamics in MDMO-PPV:PCBM blend films was studied using an approach based on the time-resolved microwave conductivity (TRMC) technique.

The electron diffusion constant clearly increases with $W_{P C B M}$, which is explained by an increased hopping rate and/or improved percolation at higher $W_{P C B M}$. The electron mobilities corresponding to these values of the electron diffusion coefficient are in good agreement with values reported in literature. A further increase to $W_{P C B M}=0.75$ leads to a reduction of the diffusion constant. The latter effect is likely due to energetic relaxation of electrons in PCBM clusters, which are formed at high PCBM content.

Although the electron diffusion coefficient at $W_{P C B M}$ $=0.75$ is relatively low, the long time for decay $(>1.4$ $\times 10^{-4} \mathrm{~s}$ ) leads to a diffusion length exceeding the typical blend layer thickness of $100 \mathrm{~nm}$ used in photovoltaic devices. Apparently, PCBM clusters in blends with a $W_{P C B M}$ $=0.75$ serve as domains in which the electrons can escape from recombination. This finding provides an additional explanation for the high PCBM content needed for optimum device performance. 


\section{ACKNOWLEDGMENTS}

This work is part of the program Polymer Photovoltaics (DPI 323) of the Dutch Polymer Institute. We thank Jörgen Sweelssen and Marc Koetse (TNO Eindhoven) for providing MDMO-PPV. The work of P.A.C.Q. forms part of the research program of the Dutch Polymer Institute (DPI).

\section{APPENDIX}

In this appendix, the analytic expression used to calculate the time dependence of electron injection from the polymer:PCBM blend into the $\mathrm{TiO}_{2}$ layer [Eq. (4)] is derived. Consider one-dimensional diffusion of a space- and timedependent charge distribution $C(x, t)$ over a length $L$ subject to the following boundary conditions:

$$
\begin{aligned}
& \left.\frac{\partial C(x, t)}{\partial x}\right|_{x=0}=0, \\
& C(x=L, t)=0 .
\end{aligned}
$$

This indicates that there is no charge flux across the front side $(x=0)$, corresponding to a fully reflecting blend/gas interface, and no charge at the blend/ $\mathrm{TiO}_{2}$ interface $(x=L)$, reflecting interfacial electron injection into $\mathrm{TiO}_{2}$. The charge distribution is written as an integral over basis functions $C_{q}(x, t)$,

$$
\begin{gathered}
C(x, t)=\int_{0}^{L}\left[f(q) C_{q}(x, t)\right] d q, \\
C_{q}(x, t=0)=\delta(x-q),
\end{gathered}
$$

such that the initial (i.e., at $t=0$ ) distribution of charges is given by $f(x)=C(x, t=0)$. The charge distributions $C_{q}(x, t)$ and $C(x, t)$ obey the diffusion equation

$$
\frac{\partial C(x, t)}{\partial t}=D_{e} \frac{\partial^{2} C(x, t)}{\partial x^{2}},
$$

with $D_{e}$ the diffusion coefficient. This equation, with the boundary conditions (A1) and initial condition (A2), is solved by

$$
\begin{gathered}
C_{q}(x, t)=\frac{1}{2 L} \sum_{k=-\infty}^{\infty} P_{k}(x) Q_{k}(q) R_{k}(t), \\
P_{k}(x)=\cos \left(\frac{\pi k x}{2 L}\right)-\cos \left[\frac{\pi k}{2 L}(x-2 L)\right], \\
Q_{k}(q)=\cos \left(\frac{\pi k q}{2 L}\right), \\
R_{k}(t)=e^{-(\pi k / 2 L)^{2} D_{e} t},
\end{gathered}
$$

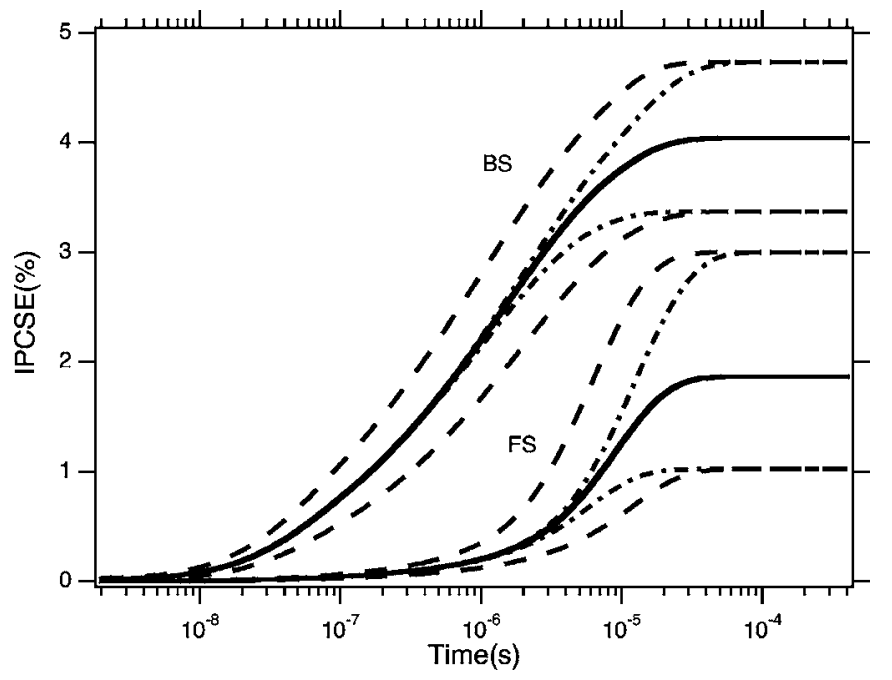

FIG. 5. Effect of parameter values on IPCSE transients calculated with Eq. (5). The full lines are obtained with parameter values from fits to experimental data [MDMO-PPV:PCBM $\left.{ }_{0.50}\left(\mathrm{TiO}_{2}\right)\right]$ on BS excitation (upper curve) or FS excitation (lower curve). The effect of a two times higher $D_{e}\left(D_{e}=7 \times 10^{-10} \mathrm{~m}^{2} / \mathrm{V} \mathrm{s}\right)$ and a two times lower $D_{e}\left(D_{e}=1.8 \times 10^{-10} \mathrm{~m}^{2} / \mathrm{V} \mathrm{s}\right)$ is demonstrated by the dashed curves. In addition, the effect of a two times longer $\tau_{e}\left(\tau_{e}\right.$ $\left.=2.2 \times 10^{-5} \mathrm{~s}\right)$ and a two times shorter $\tau_{e}\left(\tau_{e}=0.6 \times 10^{-5} \mathrm{~s}\right)$ is shown (dot dash).

as can be verified by direct substitution. The signal measured using the TRMC technique is determined by the flux through the blend/ $\mathrm{TiO}_{2}$ interface $(x=L)$ integrated over time:

$$
N(t)=-\left.D_{e} \int_{0}^{t} \frac{\partial C(x, \tau)}{\partial x}\right|_{x=L} e^{-\tau / \tau_{e}} d \tau
$$

where the exponential has been added in order to account for the decay of electrons by trapping or recombination with a rate $1 / \tau_{e}$. For both front side and back side illumination photoexcitations, the initial charge distribution can be written as

$$
f(x)=\frac{m \alpha e^{-m \alpha x}}{1-e^{-m \alpha L}}
$$

such that front side illumination photoexcitation corresponds to $m=+1$ and back side illumination photoexcitation to $m$ $=-1$.

Now one has all the ingredients to perform the double integration over space [Eq. (A2)] and time [Eq. (A5)]. The result reads 


$$
\begin{aligned}
N(t) & =-\left.\frac{D_{e}}{2 L} \sum_{k=-\infty}^{\infty} \frac{d P_{k}(x)}{d x}\right|_{x=L} \int_{0}^{L} f(q) Q_{k}(q) d q \int_{0}^{t} R_{k}(\tau) e^{-\tau / \tau_{e}} d \tau \\
& =\sum_{k=-\infty}^{\infty} \frac{\pi k D_{e}}{2 L^{2}} \sin \left(\frac{\pi k}{2}\right) \frac{m \alpha+\frac{\pi k}{2 L} e^{-m \alpha L} \sin \left(\frac{\pi k}{2}\right)}{\alpha^{2}+\left(\frac{\pi k}{2 L}\right)^{2}} \frac{m \alpha}{1-e^{-m \alpha L}} \frac{1-e^{-\left[1 / \tau_{e}+(\pi k / 2 L)^{2} D_{e}\right] t}}{1 / \tau_{e}+\left(\frac{\pi k}{2 L}\right)^{2} D_{e}} \\
& =\left(\frac{2}{1-e^{-m \alpha L}}\right) \sum_{k=0}^{\infty}\left\{\frac{\left(\frac{(-1)^{k}}{\pi(k+1 / 2)}+\frac{e^{-m \alpha L}}{m \alpha L}\right)\left(1-e^{-\left\{1 / \tau_{e}+[\pi(k+1 / 2) / L]^{2} D_{e}\right\} t}\right)}{\left.1+\left(\frac{\pi(k+1 / 2)}{m \alpha L}\right)^{2}\right]}\left[1+\frac{1}{D_{e} \tau_{e}}\left(\frac{L}{\pi(k+1 / 2)}\right)^{2}\right]\right.
\end{aligned}
$$

This is Eq. (4).

Numerical evaluation of Eq. (4) was performed by taking the summation over the first $50 k$ values. For the purpose of comparison with the experimental IPCSE transients, the simulated transients were convoluted with the $18 \mathrm{~ns}$ response time of the microwave resonant cavity used. The fact that with such a convolution the complete temporal shape of the experimental transients can be reproduced confirms that the fast initial rise is indeed determined by the response time of the cavity.

The data in Fig. 5 illustrate the effect of variation of the values of the fit parameters on the calculated IPCSE. The data show that variation of $D_{e}$ or $\tau_{e}$ by a factor of 2 leads to significant changes, which are much larger than the noise in the experimental IPCSE data. From comparison of the experimental data and the calculated data for different values of $D_{e}$ and $\tau_{e}$, the uncertainty in the values of the fit parameters is inferred to be $\sim 20 \%$.
*Corresponding author. Electronic address: t.j.savenije@tudelft.nl

${ }^{1}$ N. S. Sariciftci, L. Smilowitz, A. J. Heeger, and F. Wudl, Science 258, 1474 (1992).

${ }^{2}$ N. S. Sariciftci, D. Braun, C. Zhang, V. I. Srdanov, A. J. Heeger, G. Stucky, and F. Wudl, Appl. Phys. Lett. 62, 585 (1993).

${ }^{3}$ J. J. M. Halls, K. Pichler, R. H. Friend, S. C. Moratti, and A. B. Holmes, Appl. Phys. Lett. 68, 3120 (1996).

${ }^{4}$ S. E. Shaheen, C. J. Brabec, N. S. Sariciftci, F. Padinger, T. Fromherz, and J. C. Hummelen, Appl. Phys. Lett. 78, 841 (2001).

${ }^{5}$ T. Martens, J. D’Haen, T. Munters, Z. Beelen, L. Goris, J. Manca, M. D'Olieslaeger, D. Vanderzande, L. De Schepper, and R. Andriessen, Synth. Met. 138, 243 (2003).

${ }^{6}$ T. Martens, T. Munters, L. Goris, J. D’Haen, K. Schouteden, M. D'Olieslaeger, L. Lutsen, D. Vanderzande, W. Geens, J. Poortmans, L. De Schepper, and J. V. Manca, Appl. Phys. A: Mater. Sci. Process. 79, 27 (2004).

${ }^{7}$ H. Hoppe, M. Niggemann, C. Winder, J. Kraut, R. Hiesgen, A. Hinsch, D. Meissner, and N. S. Sariciftci, Adv. Funct. Mater. 14, 1005 (2004).

${ }^{8}$ J. K. J. van Duren, X. N. Yang, J. Loos, C. W. T. Bulle-Lieuwma, A. B. Sieval, J. C. Hummelen, and R. A. J. Janssen, Adv. Funct. Mater. 14, 425 (2004).

${ }^{9}$ C. J. Brabec, Sol. Energy Mater. Sol. Cells 83, 273 (2004).

${ }^{10}$ E. C. Chang, C. I. Chao, and R. H. Lee, J. Appl. Polym. Sci. 101, 1919 (2006).

${ }^{11}$ C. R. McNeill and P. C. Dastoor, J. Appl. Phys. 99, 033502 (2006).
${ }^{12}$ C. Melzer, E. J. Koop, V. D. Mihailetchi, and P. W. M. Blom, Adv. Funct. Mater. 14, 865 (2004).

${ }^{13}$ V. D. Mihailetchi, L. J. A. Koster, P. W. M. Blom, C. Melzer, B. de Boer, J. K. J. van Duren, and R. A. J. Janssen, Adv. Funct. Mater. 15, 795 (2005).

${ }^{14}$ V. D. Mihailetchi, L. J. A. Koster, J. C. Hummelen, and P. W. M. Blom, Phys. Rev. Lett. 93, 216601 (2004).

${ }^{15}$ V. D. Mihailetchi, J. K. J. van Duren, P. W. M. Blom, J. C. Hummelen, R. A. J. Janssen, J. M. Kroon, M. T. Rispens, W. J. H. Verhees, and M. M. Wienk, Adv. Funct. Mater. 13, 43 (2003).

${ }^{16}$ T. Offermans, S. C. J. Meskers, and R. A. J. Janssen, J. Chem. Phys. 119, 10924 (2003).

${ }^{17}$ P. A. C. Quist, T. Martens, J. V. Manca, T. J. Savenije, and L. D. A. Siebbeles, Sol. Energy Mater. Sol. Cells 90, 362 (2006).

${ }^{18}$ T. J. Savenije, J. E. Kroeze, M. M. Wienk, J. M. Kroon, and J. M. Warman, Phys. Rev. B 69, 155205 (2004).

${ }^{19}$ T. J. Savenije, J. E. Kroeze, X. N. Yang, and J. Loos, Adv. Funct. Mater. 15, 1260 (2005).

${ }^{20}$ G. Dennler, A. J. Mozer, G. Juska, A. Pivrikas, R. Osterbacka, A. Fuchsbauer, and N. S. Sariciftci, Org. Electron. 7, 229 (2006).

${ }^{21}$ D. Chirvase, J. Parisi, J. C. Hummelen, and V. Dyakonov, Nanotechnology 15, 1317 (2004).

${ }^{22}$ A. F. Nogueira, I. Montanari, J. Nelson, J. R. Durrant, C. Winder, and N. S. Sariciftci, J. Phys. Chem. B 107, 1567 (2003).

${ }^{23}$ S. A. Choulis, J. Nelson, Y. Kim, D. Poplavskyy, T. Kreouzis, J. R. Durrant, and D. D. C. Bradley, Appl. Phys. Lett. 83, 3812 
(2003)

${ }^{24}$ C. Brabec and N. S. Sariciftci, in Semiconducting Polymers, edited by G. Hadziioannou and P. F. Van Hutten (Wiley-VCH, Weinheim, 2000), p. 515.

${ }^{25}$ M. Gratzel, Nature (London) 414, 338 (2001).

${ }^{26}$ V. D. Mihailetchi, P. W. M. Blom, J. C. Hummelen, and M. T. Rispens, J. Appl. Phys. 94, 6849 (2003).

${ }^{27}$ F. Louwet, D. Vanderzande, J. Gelan, and J. Mullens, Macromolecules 28, 1330 (1995).
${ }^{28}$ J. E. Kroeze, T. J. Savenije, M. J. W. Vermeulen, and J. M. Warman, J. Phys. Chem. B 107, 7696 (2003).

${ }^{29}$ T. J. Savenije, M. J. W. Vermeulen, M. P. de Haas, and J. M. Warman, Sol. Energy Mater. Sol. Cells 61, 9 (2000).

${ }^{30}$ J. E. Kroeze, T. J. Savenije, and J. M. Warman, J. Photochem. Photobiol., A 148, 49 (2002).

${ }^{31}$ S. M. Tuladhar, D. Poplavskyy, S. A. Choulis, J. R. Durrant, D. D. C. Bradley, and J. Nelson, Adv. Funct. Mater. 15, 1171 (2005). 